\title{
Patient Specific Vascular Benchtop Models for Development and Validation of Medical Devices for Minimally Invasive Procedures
}

\author{
Maryna Kvasnytsia ${ }^{\mathrm{a}}$, Nele Famaey ${ }^{\mathrm{b}}$, Michal Böhm ${ }^{\mathrm{c}}$, Eva Verhoelst ${ }^{\mathrm{a}}$ \\ ${ }^{a}$ Materialise NV, Technologielaan 15, 3001 Leuven, Belgium \\ E-mail: Eefje.Verhoelst@materialise.be \\ ${ }^{b}$ KU Leuven, Biomechanics Section, Celestijnenlaan 300C, 3001 Heverlee, Belgium \\ 'Materialise Czech Republic, Predlicka 460/22, 40002 Usti nad Labem, Czech Republic
}

\begin{abstract}
Using realistic benchtop models in early stages of device development can reduce time and efforts necessary to move the device to further testing. In this study, we propose several patient specific vascular benchtop models for the development and validation of a robotic catheter for transcatheter aortic valve implantation. The design and manufacturing of these models, and their properties are presented. Additionally, it is demonstrated that the described design process provides virtual models that are accurately linked to the physical models.
\end{abstract}

Keywords: 3D printing, cardiovascular, HeartPrint ${ }^{\mathrm{TM}}$ Flex, silicon, material characterization, bench testing.

\section{Introduction}

Severe aortic stenosis occurs in $3.4 \%$ of the general elderly ( $>=75$ years) western population (European countries and North America) ( $\mathrm{n}=9723$ subjects) [1]. In the US alone, about 85000 aortic valve replacements are performed annually [2]. Surgical aortic valve replacement (AVR) is the current standard of care, but it has been estimated that between $30 \%$ and $60 \%$ of patients do not undergo AVR, owing to advanced age, left ventricular dysfunction, or the presence of multiple coexisting conditions. Since the introduction of TAVI, these patients are offered an alternative $[3,4,5]$. About 290000 elderly patients with severe aortic stenosis are transcatheter aortic valve implantation (TAVI) candidates, under the current indications [1].

Despite good results in a highly selected patient population, TAVI remains a delicate procedure. Interventionalists are facing several difficulties limiting valve deployment quality, and causing problems that might affect the patient's health, such as difficult visualization of the valve, limited amount of control during distant steering of the guidewire and the delivery of the valve $[2,6]$.

A European research project, "CASCADE", Cognitive AutonomouS CAtheter operating in Dynamic Environments, has been set up in order to advance the treatment of cardiovascular diseases by providing a new dexterous and intelligent (self-aware, self-exploring) robotic instrument, initially focusing on endovascular aortic valve replacement. The project aims to develop a unified control framework for continuum robots that operate in complex and deformable environments in the particular case of TAVI procedures.

During the development of new medical devices, such as robotic catheters as envisioned in the CASCADE project, various tests are required at consecutive stages of the product development lifecycle (Figure 1), including the evaluation of overall device performance, the characterization of its physical properties, and the characterization of the device interaction with living tissues. All evaluation and testing is subdivided in several stages. After invention and prototyping of the device, pre-clinical tests are performed. Those can be laboratory tests, benchtop model tests, animal studies and/or computer simulations [7]. After the pre-clinical testing phase, the device goes into a clinical testing phase in which clinical trials are organized. Based on the results of these clinical trials, a regulatory decision is made, and the product can be launched. Once on the market, the device is still monitored for its performance [8].

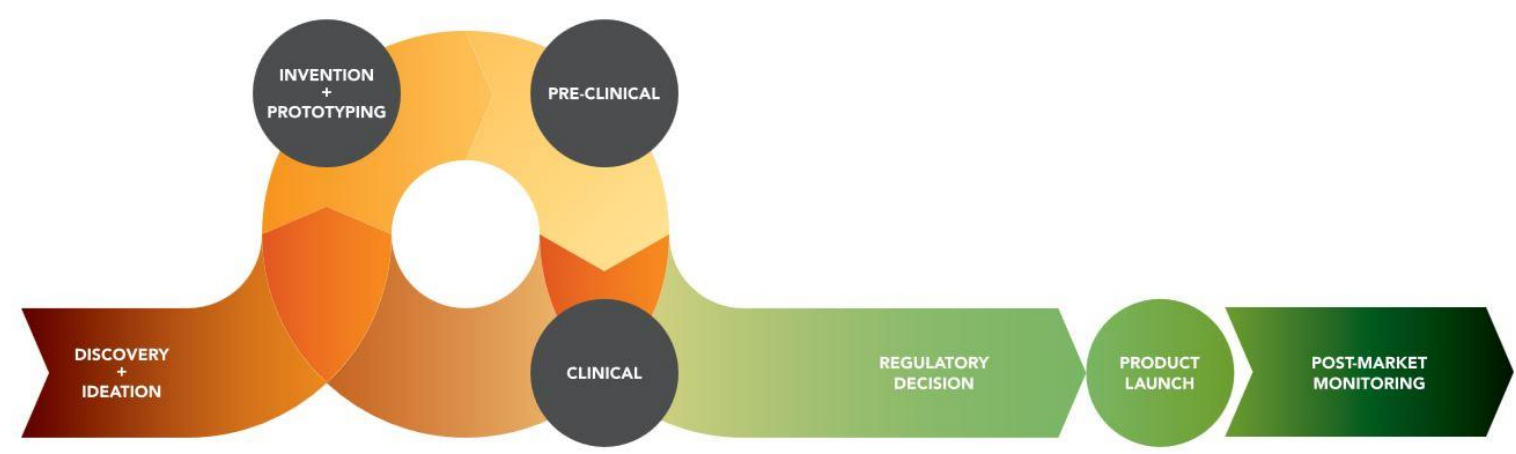

Fig. 1. New medical device development stages according to the FDA [8]. 
In the preclinical testing phase, first, biocompatibility tests are performed to evaluate the tendency of the device to cause damage to living tissues. Later on in the development cycle (Figure 1), the device is tested in live animals (animal study) which partially can predict the device behavior in humans depending on the validity of the animal model [9]. When entering the clinical testing phase the device is evaluated in human patients (clinical trial) to determine the ability of the device to perform its intended use, and to evaluate safety and efficacy (device performance). The FDA now encourages using benchtop models rather than animal models in the pre-clinical phase while developing and commercializing medical devices in an attempt to reduce costs, ethical concerns, and reliability of the testing results [7]. Animal models can differ significantly in anatomy from humans. For clinical device design, correct anatomy, size and accessibility to the site during intervention are important aspects in testing. Therefore, man-made replicas of patient-specific human anatomy used as benchtop models in the preclinical testing phase, will increase safety and efficacy before proceeding to clinical trials [10].

Animal studies and benchtop model testing represent a special type of tests known as simulated use testing. The motivation for this particular name is the fact that the animal is a simulation of the actual use (human) environment. Other forms of models for simulation testing include cadavers (both human and animal) and computational models [8]. The use of computer modeling has the potential to streamline the design, assessment and evaluation of medical devices. In addition, those computational models can make clinical trials more efficient by focusing on the most critical parameters in determining safety and effectiveness. Using both virtual models and realistic benchtop models in the early phases of device development can significantly reduce time and minimize efforts necessary to move the device to animal or cadaver testing stages. It is important to maintain a link between the physical and computational models, since physical models can represent a source of reproducible "real world data" for validation of the computational model [11].

The requirements for the ideal model to be used as substitute for a live animal, an animal cadaver, or a human cadaver in the testing of devices are quite complex. They comprise realistic geometry with a high level of anatomical detail, and similarity of physical properties (mechanical, electrical, electromagnetic, thermal, chemical, etc...) to those of humans. The models should be designed to work at body temperature in the presence of fluids. For some applications, the model needs to have a high level of transparency to allow visual or camera tracking of the device location in the model. Additionally, some experimental setups require the models to be compatible with X-Ray, MRI, ultrasound or different imaging methods.

The geometric data needed for fabrication is typically obtained in two ways. The traditional approach is to obtain data from literature on morphology, or to obtain data from cadaver measurements. Since such measurements are performed manually this method is time-consuming and permits a large degree of error. A preferable method is to obtain the geometric data directly from one or several patients by means of scanning with consequent automated virtual measurements.

After collecting the appropriate geometric data, the model components may be molded, extruded, or machined. The materials used for benchtop models development include hydrogel, silicon rubber, natural rubber, acrylic polymers, ceramics, cements, wood, styrofoam, metal, actual human tissues, actual animal tissues, and any combination thereof. For complex geometries, traditional manufacturing techniques may become cumbersome and expensive. In these cases rapid prototyping techniques offer an alternative. For some complex anatomies researchers combine 3D printing and other methods such as dip-spinning, vacuum-casting, and injection molding [12-17].

Most of the cardiovascular benchtop models present in the market are silicon models with fixed geometry. Customization of the models often requires significant costs. A non-exhaustive list of benchtop models manufacturers includes Elastrat, UnitedBiologics, BDC Laboratories, Fain Biomedical, Biomedical Modeling, The Chamberlain group, and SynDaver Labs. The soft tissue material used for the SynDaver Labs models is claimed to be almost identical to an actual human aorta. As a drawback, the material must be stored in a solution to preserve its longevity. Customization is possible, although a complete patient specific model is not possible in the soft tissue material. Moreover, those models are not transparent [15]. As far as our knowledge reaches, no information is disclosed about the material characterization of the commercially used materials for benchtop models and the comparison with real arterial tissue.

In this study, different patient specific benchtop models are designed and manufactured in different materials. Their suitability for use in both a development and a testing environment for robotic catheterization devices under investigation in the CASCADE project is evaluated. The mechanical properties of the deformable model's materials are assessed quantitatively, and compared to the ones of aortic tissue.

\section{Materials and Methods}

The robotic catheters developed and tested in CASCADE are described by Vander Poorten $e$ al. in the general CASCADE paper of this special issue, and by Devreker et al. (2014) [18]. To validate the catheter developments and the associated algorithms for steering and control, benchtop models were created. The requirements for these models were the following: (1) accurate vessel anatomy, (2) realistic mechanical properties resulting in similar 
behavior of both the catheter and the vessel in real situations, (3) the possibility to simulate risk prone areas such as calcifications, (4) compatibility with novel catheters and sensors such as intravascular ultrasound sensors, (5) transparency to allow for visual tracking of the catheter, and (6) robustness for several testing rounds. Combining all these requirements in one model was not possible. Therefore, three types of patient specific models, for different stages in the testing process, were designed and printed, namely a rigid one for use in a static testing environment, and two different deformable ones for use in a dynamic testing environment.

The static environment allowed for testing different algorithms developed in CASCADE necessary for navigation of the catheter, namely:

1. catheter shape estimation algorithm: a spline-based shape estimation interpolating shape between multiple electromagnetic (EM) sensors

2. automatic registration algorithms between pre-operative data and intra-operative data based on both force and position (electromagnetic sensor) data

3. automatic catheter steering algorithms based on a minimum-energy model

4. localization of the catheter based on flow sensing

5. catheter shape modeling based on machine learning

6. automatic catheter steering based on machine learning

7. catheter teleoperation and advanced guidance experiments

8. semi-automatic catheter steering via automatic centerline following by the tip with manual insertion/rotation

For more information on the algorithms and their validation, we refer to the general CASCADE paper by Vander Poorten et al. in this special issue. All this algorithm testing was done in a rigid benchtop model. Aside from its correct and realistic anatomy, the other main requirement for this model was its transparency. This allowed for monitoring the catheter movement by a vision system during validation of the algorithms.

Once the algorithms tested in the static environment were stabilized, the realistic behavior of the material was of higher importance compared to the transparency. The geometric correct vessel model captured only partially the necessary information about the interaction between the vessel and the catheter as it did not provide information about the forces that are exerted. Therefore, in a next stage, testing was performed in a dynamic environment simulating the real situation during TAVI. As a consequence, the benchtop model had to be deformable, with mechanical properties as close as possible to the real situation. Hence, two types of deformable models were designed and printed in an attempt to combine several requirements at once for testing of the above algorithms in a dynamic environment, and for testing two extra algorithms, namely:

1. simultaneous catheter and environment modeling: 3D reconstruction based on intravascular ultrasound (IVUS) sensing, and EM sensors

2. detection of side branches and anatomic landmarks based on IVUS

As for the above algorithms, more information can be found in the general CASCADE paper by Vander Poorten et al. in this special issue.

In what follows, the model that is designed and printed for use during static testing is called the 'rigid' model. The second and third model, which are used during the later stages of testing in a dynamic environment, are called the 'HeartPrint ${ }^{\mathrm{TM}}$ Flex' model (non-transparent), and the 'silicon' model (transparent). The models are scaled 1:1 with the real size of the patient case, and have the following length dimensions: $24 \mathrm{~cm}$ (rigid model), $62 \mathrm{~cm}$ (HeartPrint ${ }^{\mathrm{TM}}$ Flex model), and $40 \mathrm{~cm}$ (silicon model).

\subsection{Image based design of benchtop models}

Developing and testing the robotic catheterization for TAVI involved the following anatomical regions: the aortic root, the aortic arch and the ascending and descending aorta with the main branches, in which most of the interventional procedures take place. Based on a CT scan (resolution of 512x512x1179, with pixel size $0.5 \mathrm{~mm}$ ) of one case (courtesy of Dr. Herbert De Praetere, UZ Leuven), the Mimics Innovation Suite ${ }^{\circledR}$ (Materialise, Leuven, Belgium) was used to obtain the virtual 3D reconstructed aortic model, and to prepare it in a printable format. Using a graph cut based segmentation tool, the entire vasculature was segmented. Semiautomatic tools such as region grow and multislice mask edit were used to clean up the vessel mask (Figure 2). The graph cut based segmentation process used for vasculature segmentation was validated on 36 patient cases (CT). On average, the difference between the automatic result and the ground truth was comparable to the differences found between several operators performing a segmentation (average overlap measure of 0.87 for the graph cut segmentation tool versus 0.88 for the manual segmentation). The cases not showing a good overlap with the ground truth typically had inclusion of spine bone structures in the resulting segmented model. These can be easily removed via manual editing tools [19]. Furthermore, manual segmentation tools were applied to define the region of interest for the model. The segmented vasculature was represented by a triangulated surface (STL file format). This surface mesh can be transformed to a volume mesh for later finite element analysis (FEA). In the Mimics Innovation Suite ${ }$, the stl model was hollowed to create a wall thickness for later 3D printing. Specially designed connectors were added to all models in order to connect the model to a (pumping) circulation system [20]. 


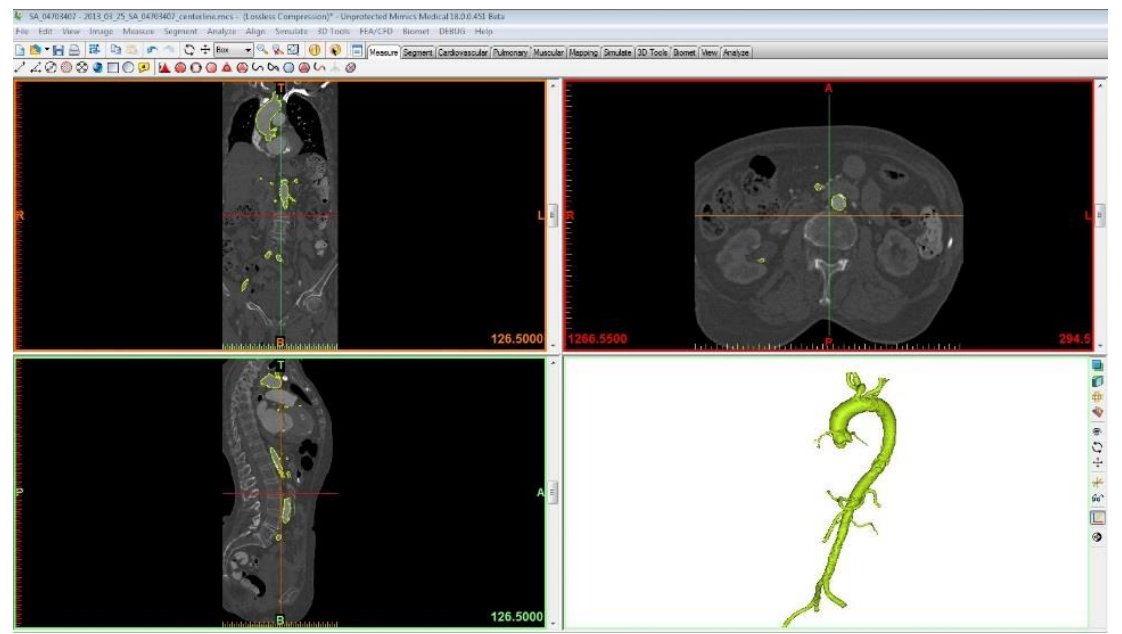

Fig. 2. CT images of one case (courtesy of Dr. Herbert De Praetere, UZ Leuven) with the vascular segmentation mask in Mimics 16.0 (Materialise NV, Belgium).

\subsection{Rigid benchtop model}

The region of the rigid model included only the aortic arch with the coronary arteries, without the aortic valve leaflets. The model was 3D printed using a transparent Tusk material (stereolitography technology). In order to provide a smooth surface, a cosmetic finish was applied to the model which imposes a requirement of minimum $2 \mathrm{~mm}$ wall thickness of the model. In order to apply this cosmetic finish from inside the model, it was printed in two parts and then glued. Material properties of the material were not measured quantitatively as these were anyway not realistic. The transparency of the rigid model was very high, making it well suited for catheter tracking based on a vision system (Figure 3).

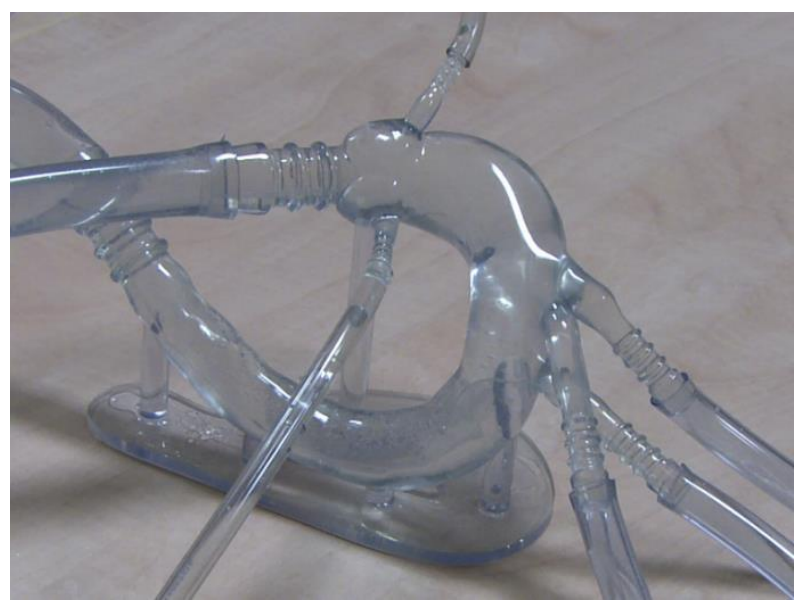

Fig. 3. The highly transparent 3D printed rigid benchtop model with tubes from the testing environment attached.

\subsection{Deformable HeartPrint ${ }^{T M}$ Flex benchtop model}

A TAVI procedure deals with the positioning of an aortic valve in a dynamic environment (the aortic annulus of the beating heart and the aorta surrounded by lungs).Therefore, the developed catheter and associated algorithms should be tested in such a dynamic environment. This was created by designing and printing deformable models which were connected to a pumping circuit to simulate the dynamics of the aorta during the procedure. As a consequence, the model needed to have mechanical properties close to those of real arterial tissue. This was assessed both qualitatively through inspection by experts (similar behavior but not identical), and quantitatively (see below). A media file is available showing the deformation of the benchtop model incorporated into the pumping circuit. 
The deformable model was based on the same images as the rigid model, including a wider range of anatomy: the aortic valve with leaflets, left and right coronary artery, ascending aorta, arch, brachiocephalic artery, left common carotid artery, left subclavian artery, descending and abdominal aorta, mesenteric artery, left and right renal, celiac trunk, left and right common iliac arteries. The deformable model was printed in HeartPrint ${ }^{\mathrm{TM}}$ Flex with an Objet500 Connex printer (Stratasys) (Figure 4). Biglino et al. (2013) found that the HeartPrint ${ }^{\mathrm{TM}}$ Flex material can be used to print compliant models with properties that are within the range of human arterial tissue properties, namely a distensibility of $2.2 \times 10^{-3}$ to $7.3 \times 10^{-3} \mathrm{mmHg}^{-1}$ described in literature [20, 21]. The distensibility for the HeartPrint ${ }^{\mathrm{TM}}$ Flex sample of $1.5 \mathrm{~mm}$ thickness is measured to be $3.0 \times 10^{-3} \mathrm{mmHg}^{-1}$. For this reason, the wall thickness of the deformable model was set to $1.5 \mathrm{~mm}$ as this material thickness guarantees realistic mechanical properties of the model [20].

The deformable model was split into 4 parts to create a modular test-bed structure. Modularity allowed more flexibility in terms of redesign of different anatomy types, and additionally limited the costs in case a particular part would be damaged as it would only require rebuilding a part of the model. The main drawback of the HeartPrint ${ }^{\mathrm{TM}}$ Flex material is its rupture sensitivity. The parts that were selected are:

- Part 1: the aortic valve plus coronaries;

- Part 2: the ventricular space;

- Part 3: the arch and the thoracic aorta, at least $10 \mathrm{~cm}$ of the brachiocephalic, left common carotid, left common subclavian;

- Part 4: the abdominal aorta, iliac bifurcation including the renal, mesenteric and celiac artery.

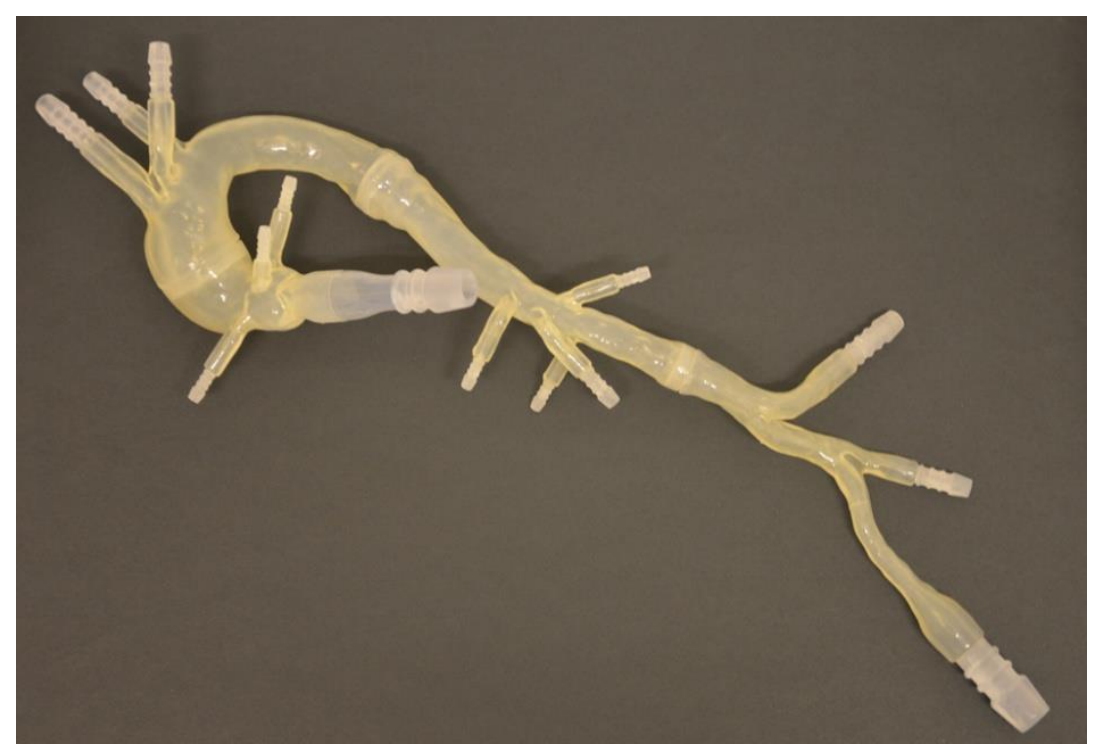

Fig. 4. The 3D printed HeartPrint ${ }^{\mathrm{TM}}$ Flex deformable benchtop model showing its modular structure consisting of 4 segments, and the set of connectors.

For the development of intelligent robotic catheters it is important to take into account the calcifications present in the vessel, which may hinder the catheter navigation or lead to embolization due to calcification rupture. The device being developed should be able to recognize calcifications to avoid risk during the procedure. Therefore, it is important to model calcifications in benchtop models for next device validation iterations. The severity and extent of mineralization can be derived from analyzing preprocessed images. The calcifications were segmented in the Mimics Innovation Suite ${ }^{\circledR}$ based on Hounsfield units (HU), and transformed to a mesh file. This method is similar to the one described by Ewe et al. (2011) [22]. The intensity values of the calcifications on the CT images allowed for a global threshold value of $800 \mathrm{HU}$ to segment them. The corresponding material properties were assigned to the vessel walls and the calcifications (Figure 5). 


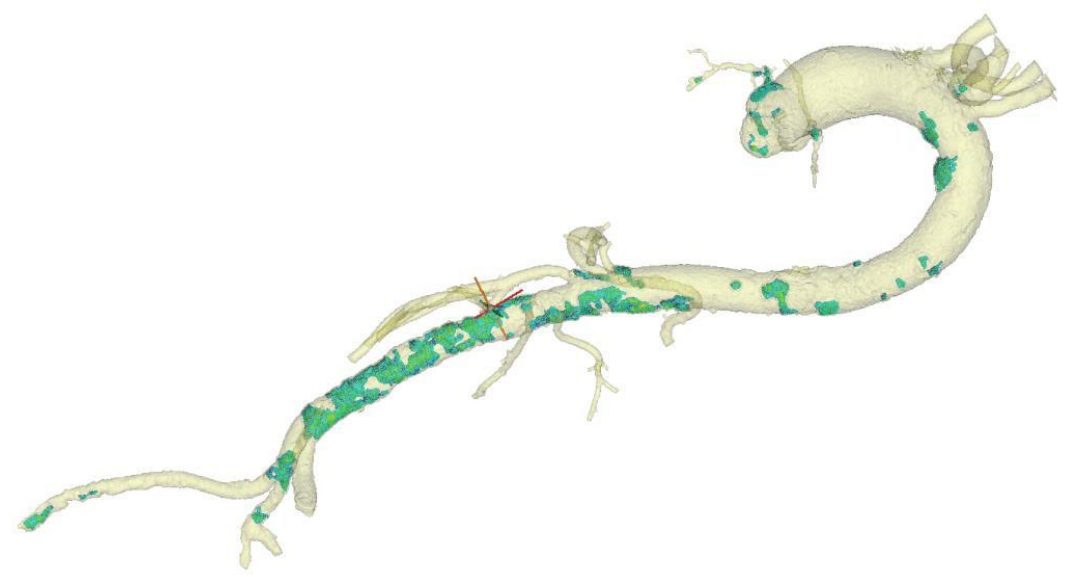

Fig. 5. Calcification distribution along the vasculature of interest assessed via image processing in Mimics 16.0 (Materialise NV, Leuven).

Multimaterial inkjet 3D printing technology provides a unique opportunity to easily introduce calcifications in the benchtop model design. Patient specific calcifications, based on the segmentations of the CT scan, were added to the deformable model. The calcifications were represented as regions printed from a rigid, opaque photopolymer (Figure 6).

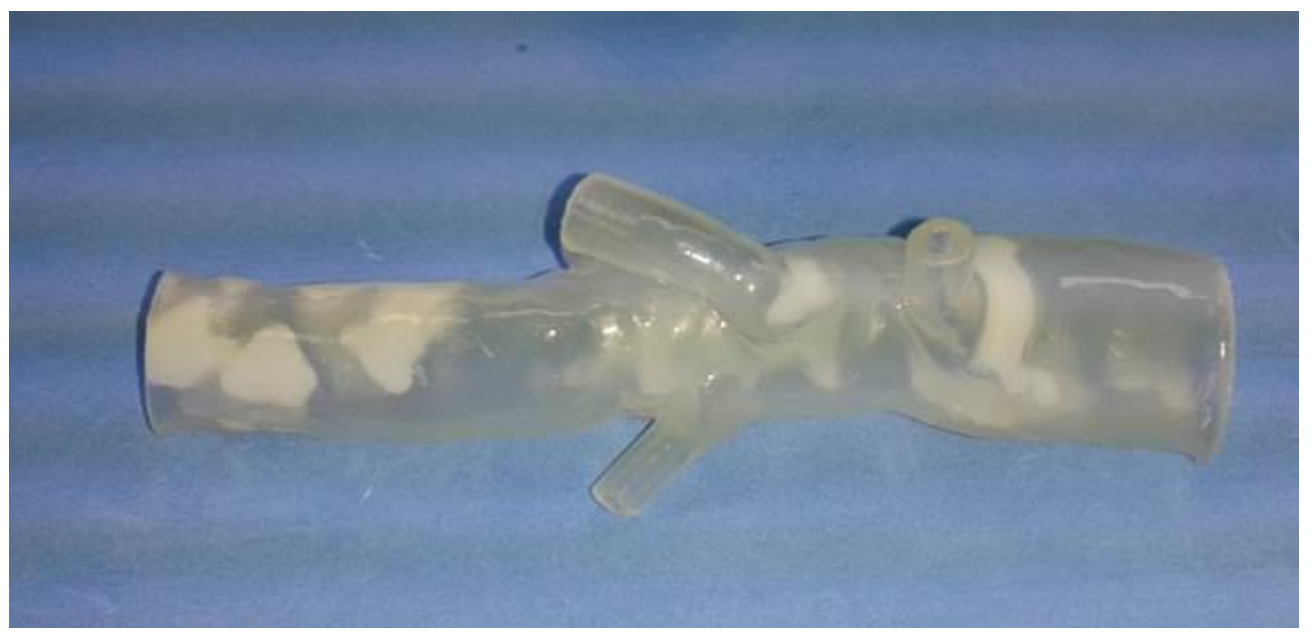

Fig. 6. 3D printed HeartPrint ${ }^{\mathrm{TM}}$ Flex model of the abdominal aorta with patient specific calcifications.

\subsection{Deformable silicon benchtop model}

Although the HeartPrint ${ }^{\mathrm{TM}}$ Flex material has a lot of benefits as described above, two issues remain, namely its limited transparency, and its rupture sensitivity. For advanced stages of validation of catheter navigation, high benchtop model transparency is needed since the validation is performed with visual tracking of the catheter within the model [23]. Such a model should be both deformable and transparent. Moreover, it should have increased robustness to withstand the load during the tests.

Silicon models, such as the ones from Elastrat, are often used in benchtop testing. The HeartPrint ${ }^{\mathrm{TM}}$ Flex material's transparency is suboptimal compared to silicon transparency. Manufacturing of a custom silicon models based on patient data is however still a challenge. In this study, a new hybrid method for patient specific silicon models manufacturing was developed. This multi-step method combines usage of the Mimics Innovation suite ${ }^{\circledR}$ for segmentation of CT data, 3D printing of patient specific shells, and vacuum casting of the silicon model. The resulting model possesses high robustness and transparency (Figure 7). 


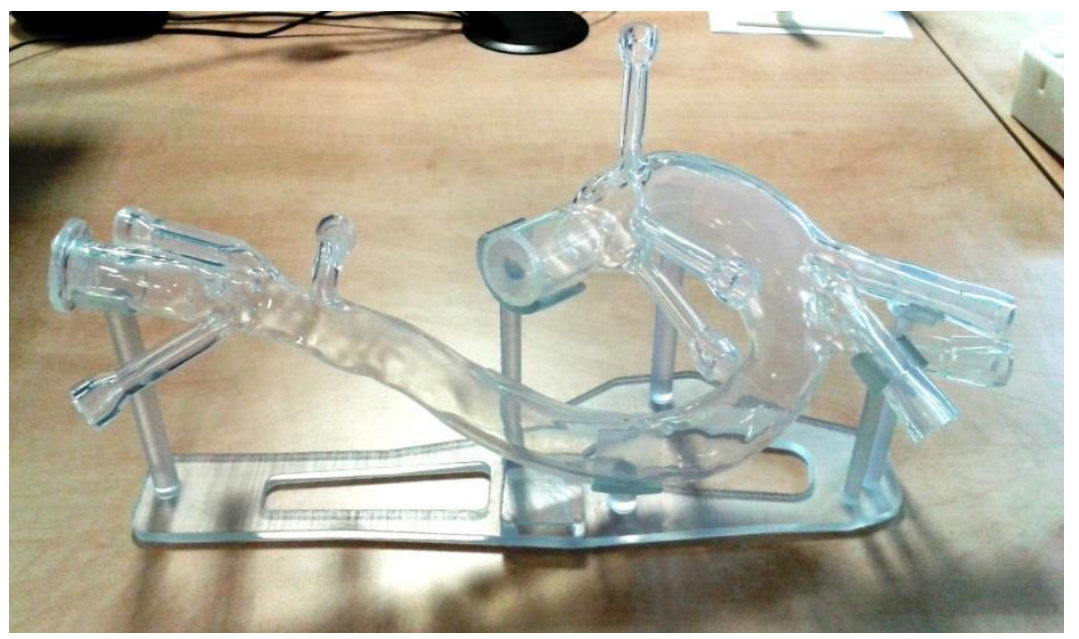

Fig. 7. Patient specific silicon benchtop model manufactured by a hybrid method. The model is placed on a 3D printed support designed to preserve the anatomical vascular orientation and avoid deformation.

\subsection{Testing of the Intravascular imaging compatibility of the deformable models}

When developing the robotic catheterization devices under investigation in the CASCADE project, the preoperative data is taken into account for navigation. Nevertheless, one cannot solely rely on this data for programming the steering of the catheter. Since the vasculature is deformable and dynamic in nature, preoperatively acquired information has limited value during the intervention. A constant update of the current anatomy during the intervention is necessary. On the other hand, the common intra-operative information acquisition method, namely angiography, is dependent on the injection of contrast agent, and is damaging as it exposes the patient to excessive radiation. In addition, information about the inside of the vessel enhances the user or controller's awareness of the catheter/vessel system which could lead to better navigation. For these reasons, it was decided to use IntraVascular Ultrasound (IVUS) sensors, as this technique allowed for both inside vessel information, and intra-operative information to improve catheter navigation. Further information on this topic can be found in the general CASCADE paper by Vander Poorten et al. in this special issue.

For development and testing of this catheter steering, the compatibility of the deformable benchtop testing models with a Visions PV $.035^{\circledR}$ (Volcano Corp., CA, USA) was assessed qualitatively. A picture of the testing set-up is shown below (figure 8). Techniques to extract the vessel wall from this noisy sensor were developed and tested on the benchtop models $[24,25]$.

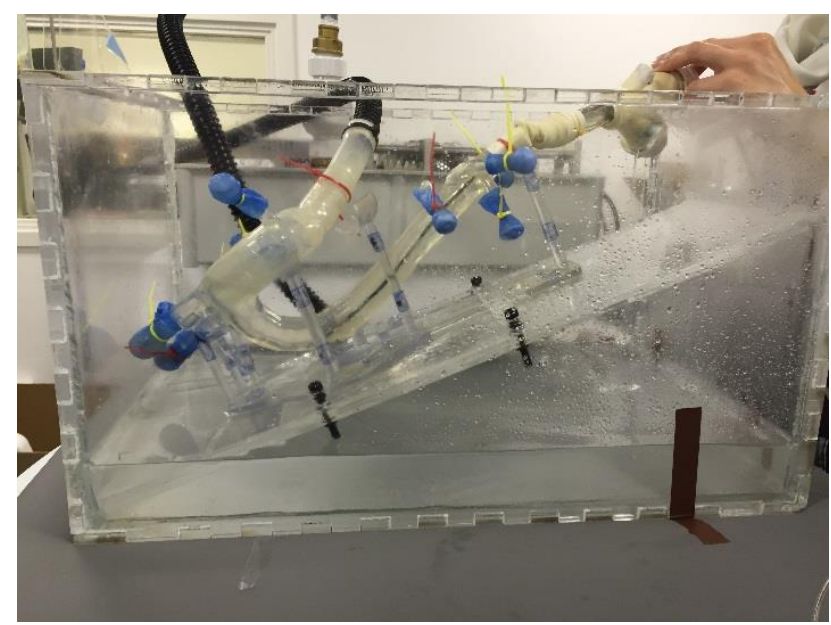

Fig. 8. Picture of the set-up where the IVUS probe was inserted in the HeartPrint ${ }^{\mathrm{TM}}$ Flex model attached to the pumping circuit. The model was tilted inside the box to enable the connection of the model with the pump, and to enable insertion of the IVUS probe. Placing the model in a box enabled submerging the region of interest in a fluid making it air-free which was necessary for IVUS testing. The blue elements closed the openings of all side branches, while the black tubes 
made the connections with the pumping circuit. The black line within the model was the IVUS probe, held by the hand on the right side of the picture. (Images provided by Stamatia Gianarou, Su-Lin Lee, Liang Zhao, Imperial College, London).

\subsection{Testing of the mechanical properties of the deformable models}

The mechanical characteristics of both deformable models were assessed quantitatively, and compared with aortic tissue mechanical characteristics. The friction inside the models was assessed qualitatively through testing by surgeons.

Planar biaxial tensile tests were performed on the HeartPrint ${ }^{\mathrm{TM}}$ Flex and silicon material on a BioTester (CellScale, Waterloo, Canada). 13×13mm square samples were mounted in the device using biorakes (Figure 9). Two samples of each material type were tested. The circumferential direction of the sample was aligned with the $\mathrm{X}$-axis of the device. A displacement-controlled protocol was performed in which the sample was elongated in the $\mathrm{X}$ - and $\mathrm{y}$-direction at a loading rate of $1 \mathrm{~mm} / \mathrm{s}$. Cyclic stretch was applied according to the following ratios $(\mathrm{X}-\mathrm{Y})$ : $20 \%-20 \% ; 10 \%-20 \%$ and $20 \%-10 \%$, with 5 cycles of each ratio. The corresponding force along each axis was acquired using a Honeywell (model 31 ) load cell with a $+/-23 \mathrm{~N}$ range $(0.2 \%$ full scale accuracy). Deformation was tracked using markers and a CCD camera (Figure 9).

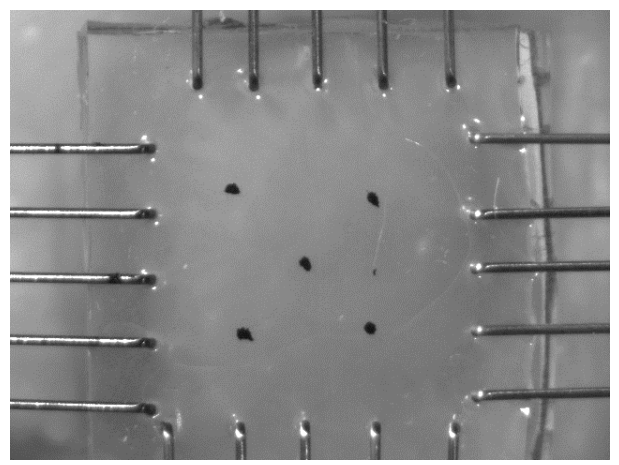

Fig. 9. Sample of a planar biaxial test on a silicone material patch. The markers are used to track the deformation gradient.

Parameter fitting to the Holzapfel-Gasser-Ogden (HGO) model [26] as well as to the Neo-Hookean material model was performed, where the experimentally measured Cauchy stresses were compared to model stresses in a nonlinear optimization process using Matlab. The experimental Cauchy stresses $\left(\sigma_{\text {exp }}\right)$ were calculated as:

$$
\sigma_{\exp }=J^{-1} F P
$$

with $F$ the deformation gradient calculated from the marker displacements, $P$ the first Piola-Kirchhoff stress calculated by dividing the measured force by the initial cross section (where the area between the rakes is considered), and $J$ the determinant of $F$.

The model stresses $\left(\sigma_{\bmod }\right)$ were obtained by calculating:

$$
\sigma_{\text {mod }}=-p \mathrm{I}+2 \frac{\partial \Psi(\mathrm{F})}{\partial \mathrm{F}} \mathrm{F}^{T}
$$

where $p$ serves as a Lagrange multiplier that can be derived from the condition $\sigma_{33}=0, \mathbf{I}$ is the identity tensor, and $\Psi$ is the strain energy density function that describes the HGO material or Neo-Hookean (NH) material [26]:

$$
\begin{gathered}
\psi_{N H}=c\left(I_{1}-3\right) \\
\psi_{H G O}=c\left(I_{1}-3\right)+\frac{k_{1}}{2 k_{2}} \sum_{i=4,6} e^{\left(k_{2}\left(\kappa I_{1}+(1-3 \kappa) I_{i}-1\right)^{2}\right)}-1
\end{gathered}
$$

with

$$
\begin{aligned}
& I_{1}=\operatorname{tr}(\boldsymbol{C}) \\
& I_{4}=\boldsymbol{M} \cdot(\boldsymbol{C M})
\end{aligned}
$$




$$
I_{6}=\boldsymbol{M}^{\prime} \cdot\left(\boldsymbol{C M}^{\prime}\right)
$$

the invariants of the right Cauchy-Green deformation tensor $\mathbf{C}=\mathbf{F}^{\mathbf{T}} \mathbf{F}$ and $\mathbf{M}$. $\mathbf{M}$ ' presents the preferred fiber directions of the two collagen fibers that are generally present in arteries. $c, k_{1}, k_{2}, \alpha$ and $\kappa$ are the material parameters to be obtained from the parameter fitting. $c[\mathrm{~Pa}]$ is related to the stiffness of the matrix material of arterial tissue, $k_{1}[\mathrm{~Pa}]$ is related to the stiffness of the collagen matrix of arterial tissue, $k_{2}[-]$ is a dimensionless parameter that expresses the stiffening effect of arterial tissue, $\alpha$ [rad] represents the angle under which the collagen fibers are oriented, and $\kappa[-]$ is a dimensionless parameter that expresses the dispersion of the collagen fibers [26].

Note that the first part of the HGO material is the same as the Neo-Hookean material, and represents the isotropic part. The second part represents the nonlinear directional stiffening effect due to the presence of collagen fibers in arteries (equation 3 and 4). For more information on these material models and other continuum-mechanical aspects, please refer to [27].

\section{Results}

All three benchtop models were tested to check their suitability for use in the testing environment for the catheter development in the CASCADE project. The compatibility with IVUS, and the mechanical properties are presented below.

\subsection{IVUS compatibility}

When testing the IVUS imaging in the models, the HeartPrint ${ }^{\mathrm{TM}}$ Flex material appeared very clearly in the ultrasound image. (Figure 10). Also, the silicon model had a satisfactory level of contrast on the IVUS images as it was possible to use image processing algorithms to extract the inner vessel wall contour (Figure 11 and 12).

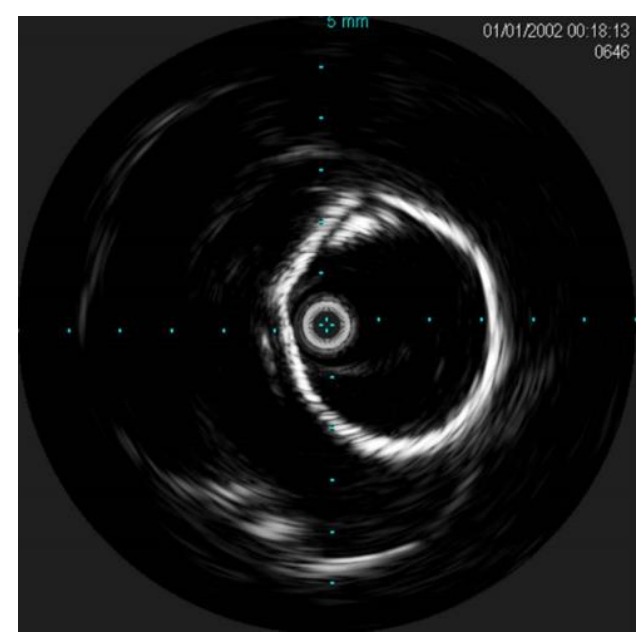

Fig. 10. IVUS image of the HeartPrint ${ }^{\mathrm{TM}}$ Flex model with embedded calcifications. No textural differences are observed between the calcifications and the vessel wall, though the calcifications can be separated by geometrical features (Images provided by Stammatia Gianarou, Su-Lin Lee, Liang Zhao, Imperial College, London). 


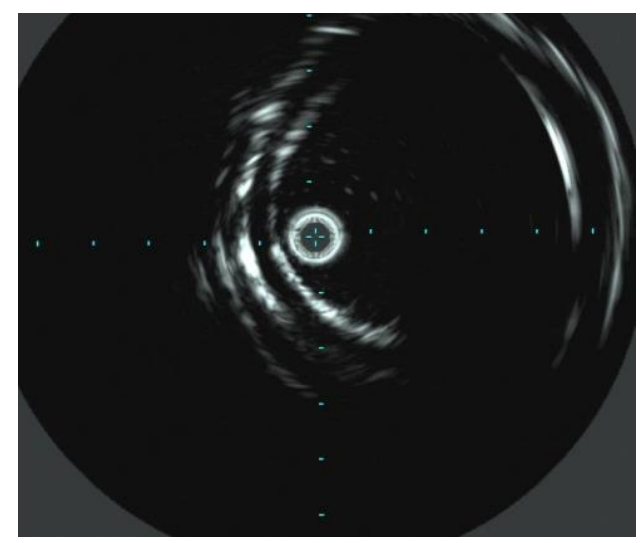

Fig. 11. IVUS image of the silicon model (Images provided by Stammatia Gianarou, Su-Lin Lee, Liang Zhao, Imperial College, London).

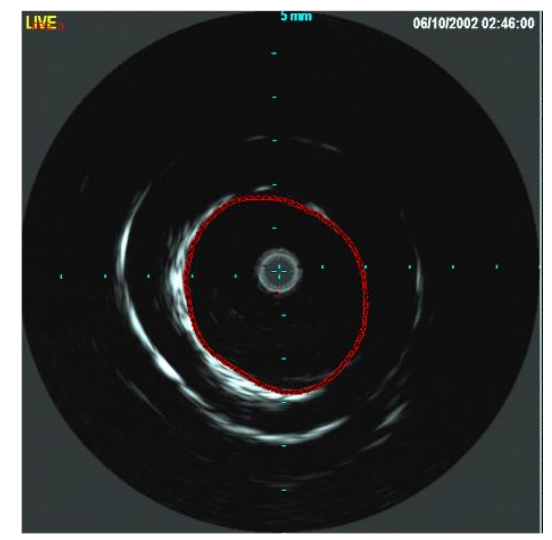

Fig. 12. IVUS image of the model with automatic detection of the inner vessel wall contour (Images provided by Stammatia Gianarou, Su-Lin Lee, Liang Zhao, Imperial College, London).

\subsection{Mechanical properties characterization}

The material properties of the rigid model were not measured quantitatively as these were not realistic. For the mechanical properties of the used materials of both deformable models, the results of the parameter fitting procedure for each of the samples are shown in table 1, along with material parameters of a typical healthy human aorta as obtained from unpublished data as collected in [28]. The relatively high variability (st. dev) of the HGO parameters of the aorta are in the same range as what is generally found when testing biological tissue [29, 30].

Table 1. Parameters of the HeartPrint ${ }^{\mathrm{TM}}$ Flex and silicon material resulting from the fitting process, as well as the corresponding parameters of a healthy human aorta. S1, S2 -silicon test sample 1, 2, HPF1, HPF 2 - HeartPrint ${ }^{\mathrm{TM}}$ Flex sample 1,2. Note that for the latter samples, parameters were sought with and without the fiber reinforcement component (respectively, with the HGO model = the first line per sample, and the NH model $=$ the second line per sample), whereas for the aortic material, which is clearly a composite, only parameters with the reinforcement component are shown. NRSME the normalized root means square error quantifying the goodness of fit; $c, k_{1}, k_{2}, \alpha$ and $\kappa$-material parameters obtained from the parameter fitting. For more information on these material models and other continuum-mechanical aspects, please refer to [27].

\begin{tabular}{|l|rrrrrrr|}
\hline & $\mathrm{c}[\mathrm{MPa}]$ & $\mathrm{k} 1[\mathrm{MPa}]$ & $\mathrm{k} 2[-]$ & \multicolumn{1}{c}{$\alpha[\mathrm{rad}]$} & $\mathrm{k}[-]$ & \multicolumn{2}{c|}{ NRSME } \\
\hline S1 & 0,15 & 0,47 & $1,40 \mathrm{E}-09$ & 1,57 & 0,32 & 0,0117 \\
& 0,18 & $/$ & $/$ & $/$ & $/$ & 0,0348 \\
S2 & 0,13 & 0,46 & $1,27 \mathrm{E}-09$ & 1,57 & 0,31 & 0,013 \\
& 0,16 & $/$ & $/$ & $/$ & $/$ & 0,0391 \\
HPF1 & 0,12 & 0,03 & 2,69 & 1,57 & 0,2 & 0,0135
\end{tabular}




\begin{tabular}{|l|rrrrrr|} 
& 0,13 & $/$ & $/$ & $/$ & $/$ & 0,0264 \\
HPF2 & 0,13 & 0,02 & $5,93 \mathrm{E}-01$ & 1,57 & 0,1 & 0,0133 \\
& 0,14 & $/$ & $/$ & $/$ & $/$ & 0,0239 \\
\hline \multirow{3}{*}{ aorta } & 0,06 & 0,27 & 13,4 & 0,79 & 0,29 & 0.0805 \\
st.dev & $\pm 0,038$ & $\pm 0,30$ & $\pm 16,8$ & $\pm 0,86$ & $\pm 0,03$ & \pm 0.0283 \\
\hline
\end{tabular}

The HeartPrint ${ }^{\mathrm{TM}}$ Flex material is slightly less stiff than the silicon, and hence approximates the native material better. Secondly, the normalized root mean square errors (NRSME) indicate a slightly better fit for the HGO material as compared to the Neo-Hookean material. This indicates a slight directional stiffening of both the silicon and the HeartPrint ${ }^{\mathrm{TM}}$ Flex material. Though still small, the nonlinearity is more pronounced and hence again closer to native arterial behavior in the HeartPrint ${ }^{\mathrm{TM}}$ Flex material. The far lower degree of nonlinear stiffening in the HeartPrint ${ }^{\mathrm{TM}}$ Flex material compared to the native aortic material is compensated by the higher shear modulus. In conclusion, the HeartPrint ${ }^{\mathrm{TM}}$ Flex material matches the overall linear stiffness behavior of the tissue relatively well, but will only slightly capture the nonlinear stiffening effects.

All models, rigid, the HeartPrint ${ }^{\mathrm{TM}}$ Flex material model and the silicon model demonstrate too high friction compared to the one of real vessel walls based on surgeons' feedback. This can be overcome by adding oil to the setup to decrease friction.

\section{Discussion}

The developed physical benchtop models provide a good representation of the clinical reality from geometrical standpoint. All are patient specific. They offer a suitable environment for testing medical devices during different stages of the development process. Each model has its own unique features desired for specific tests. By the design of connectors and support, all three models can be connected to a pumping system replicating blood flow circulation. The rigid 3D printed model offers high transparency and robustness. The material characteristics of both deformable models, and especially the HeartPrintTM Flex one, was close to the one of vascular tissue, however still some differences were present. The 3D printing process used to create the deformable model in HeartPrint ${ }^{\mathrm{TM}}$ Flex material allows incorporation of calcifications in the vessel model, which is, at the moment, not possible in case of the hybrid method for silicon models manufacturing. At the same time, silicon models demonstrate much higher robustness and transparency compared to the 3D printed models. Both deformable models are visible using IVUS.

Further work should focus on manufacturing deformable models which would combine advantages of both technologies on the way to improve realistic properties of benchtop models, considering also minimizing time and cost needed required for production.

Next to the manufacturing evaluation, the proposed method of benchtop model design has an additional benefit. In terms of the CASCADE framework, not only a physical model is used for validation of the hardware and software, but also the virtual aorta model is extensively used for the computational simulation part of the workflow, and for the algorithm's validation. The Mimics Innovation Suite ${ }^{\circledR}$ is able to provide this link between physical and virtual models to align all performed tests. There were algorithms developed to translate the pre-operative geometric data in a hex-mesh. This hex-mesh is then used as the basis of a 3D mechanical model that will be evaluated and updated through real-time Finite Element Method, FEM.

In conclusion, the proposed design and manufacturing process provides an interesting solution for different stages of medical device development as the testing environment from Materialise includes both realistic physical and virtual components with an accurate link between them. Nevertheless, there are still differences in mechanical characteristics between the benchtop models and the real aortic tissue.

\section{Acknowledgments}

The research leading to these results has received funding from the European Union's 7th Framework Program FP7-ICT No. 601021 (CASCADE).

\section{References}

[1] R.L. Osnabrugge, D. Mylotte, S.J. Head, N.M. Van Mieghem, V.T. Nkomo, C.M. LeReun, A.J. Bogers, N. Piazza, A.P. Kappetein, Aortic stenosis in the elderly: disease prevalence and number of candidates for transcatheter aortic valve replacement: a meta-analysis and modeling study. J. Am. Coll. Cardiol. 62(11) (2013), 1002-12. 
[2] J-M. Sinning, N. Werner, G. Nickenig, E. Grube, Next-generation transcatheter heart valves: current trials in Europe and the USA, Methodist Debakey Cardiovasc. J. 8(2) (2012), 9-12.

[3] A.K. Bose, J.D. Aitchison, J.H. Dark, Aortic valve replacement in octogenarians. J. Cardiothorac. Surg. 2 (2007), 33 35 .

[4] V.H. Thourani, G. Ailawadi, W.Y. Szeto, et al., Outcomes of surgical aortic valve replacement in high-risk patients: a multiinsitutional study. Ann. Thorac. Surg. 91 (2011), 49-56.

[5] S.K. Kodali, M.R. Williams, C.R. Smith, et al., Two-year outcomes after transcatheter or surgical aortic-valve replacement. N Engl. J. Med. 366 (2012), 1686-1695.

[6] B. Clayton, G. Morgan-Hughes, C. Roobottom, Transcatheter aortic valve insertion (TAVI): a review. Br. J. Radiol. 87 (2014), 1033.

[7] Guidance for Industry and FDA Staff General Considerations for Animal Studies for Cardiovascular Devices, (2010) Retrieved from http://www.fda.gov/downloads/MedicalDevices/DeviceRegulationandGuidance/GuidanceDocuments/UCM220772.pdf.

[8] Regulatory Science in FDA's Center for Devices and Radiological Health: A Vital Framework for Protecting and Promoting Public Health, (2015). Retrieved from http://www.fda.gov/downloads/AboutFDA/CentersOffices/CDRH/CDRHReports/ucm274162.pdf.

[9] V.C. Henderson, J. Kimmelman, D. Fergusson, J.M. Grimshaw, D.G. Hackam, Threaths to validity in the design and conduct of preclinical efficacy studies: a systematic review of guidelines for in vivo animal experiments. PLoS Med. 10(7) (2013).

[10] S. Kenny and E. McDermott, Simulated testing in medical device design (2006). Retrieved from http://www.creganna.com/wp-content/uploads/SimulatedTestinginMedicalDeviceDesign.pdf.

[11] C.K. Zarins, C.A. Taylor, Endovascular device design in the future: transformation from trial and error to computational design. J. Endovasc. Ther. 16(1) (2009), 12-21.

[12] L. Allard, G. Soulez, B. Chayer, Z. Qin, D. Roy, G. A. Cloutier, Multimodality vascular imaging phantom of an abdominal aortic aneurysm with a visible thrombus, Med Phys,. 40(6) (2013).

[13] K. Caute, G. N. Palafox, F. Medina, and R. B. Wicker, Complex silicone aorta models manufactured using a dip-spin coating technique and water-soluble molds, in Proc. Summer Bioengineering Conference, (2003).

[14] J.Y. Chueh, A.K. Wakhloo, M.J. Gounis, Neurovascular Modeling: Small-Batch Manufacturing of Silicone Vascular Replicas, Am. J. Neuroradiol. 30(6) (2009), 1159-64.

[15] S.R. de Galarreta, C. Aitor, R. Antón, E.A. Finol, Abdominal aortic aneurysm: from clinical imaging to realistic replicas, Biomech. Eng. 136(1) (2014).

[16] K. Knox, C. W. Kerber, S. A. Singel, M. J. Bailey, and S. G. Imbesi, Stereolithographic Vascular Replicas from CT Scans: Choosing Treatment Strategies, Teaching, and Research from Live Patient Scan Data, Am. J. Neuroradiol. 26(6) (2005),1428-1431.

[17] C. Sakezles, Synthetic Human Tissue Models Can Reduce the Cost of Device Development, Med. Device Technol. 20(1) (2009), 32-4.

[18] A. Devreker, S. Portoles Diez, A. Gijbels, B. Rosa, J. Vander Sloten, E. Vander Poorten, D. Reynaerts, H. De Praetere, P. Herijgers, Towards intuitive operation of a robotic catheter, in Proc. CRAS 2014, (Genoa, Italy, 2014), 100-103.

[19] B. Bosmans, M. Kvasnytsia, J. Maes, P. Verschueren, Mimics CT Heart tool, (2015). Retrieved from: http://biomedical.materialise.com/white-paper-mimics-ct-heart-tool-abstract .

[20] G. Biglino, P. Verschueren, R.Zegels, A. M Taylor and S. Schievano, Rapid prototyping compliant arterial phantoms for in-vitro studies and device testing, J. Cardiovasc. Magn. Reson., 15(2) (2013).

[21] M. F. O'Rourke, J.A. Staessen, C. Vlachopoulos, D. Duprez, G.E. Plante, Clinical applications of arterial stiffness; definitions and reference values, Am. J. Hypertens, 15(5) (2002): 426-444.

[22] S.H. Ewe, A.C.T. Ng, J.D. Schuijf, et al., Location and severity of aortic valve calcium and implications for aortic regurgitation after transcatheter aortic valve implantation. Am. J. Cardiol. 108(10) (2011), 1470-1477.

[23] A. Di Iasio, T. Visarius, G. Martena, M. Sette, A novel sensorized catheter for precise stenotic heart valve targeting: an application of the Medyria Trackcath Catheter Positioning System, in Proc. CRAS 2014, (Genoa, Italy, 2014), 125-129.

[24] A. Karlas, Su-Lin Lee, Vessel Retargeting using Intravascular Ultrasound, in Proc. CRAS 2014, (Genoa, Italy, 2014), 538.

[25] P-L. Chan, D. Stoyanov, Simultaneous Segmentation and Registration Using a Prior Model, in Proc. CRAS 2014, (Genoa, Italy, 2014), 21-22.

[26] T.C. Gasser, R. W. Ogden, G. Holzapfel, Hyperelastic modelling of arterial layers with distributed collagen fibre orientations. J. Roy. Soc. Interface, 3(6) (2006), 15-35.

[27] G.A. Holzapfel, Nonlinear Solid Mechanics: A Continuum Approach for Engineering (John Wiley \& Sons, 2000).

[28] M. Van den Abbeele, M. Smoljkic, H. Fehervary, S. Verleden, N. Famaey, J. Vander Sloten, Characterization of mechanical properties of human pulmonary and aortic tissue. IFMBE Proceedings (Dubrovnik, Croatia, 2014), 45, 387390.

[29] D.M. Pierce, F. Maier, H. Weisbecker, C. Viertler, P. Verbrugghe, N. Famaey, I. Fourneau, P. Herijgers and G.A. Holzapfel, Human thoracic and abdominal aortic aneurysmal tissues: damage experiments, statistical analysis and constitutive modeling. J. Mech. Behav. Biomed., 41 (2015), 92-107. 
[30] G.A. Holzapfel, G. Sommer, C.T. Gasser and P. Regitnig, Determination of layer-specific mechanical properties of human coronary arteries with non-atherosclerotic intimal thickening, and related constitutive modeling. Am. J. Physiol. Heart Circ. Physiol., 289(5) (2005), 2048-2058.

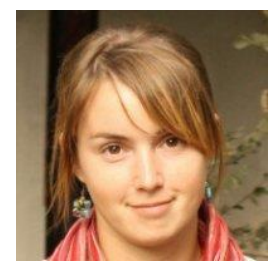

Maryna Kvasnytsia received her MSc degree in Physics and Electronics from the Taras Shevchenko National University of Kyiv, in 2006. From Year 2013 she is Research Engineer in Materialise NV.

Her research interests include medical image segmentation, registration and analysis algorithms, and biomedical engineering.

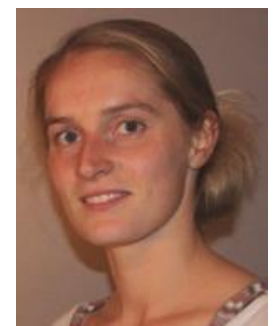

Dr. Ir. Nele Famaey received her MSc and $\mathrm{PhD}$ degree in Mechanical Engineering at KU Leuven, Belgium, in 2006 and 2012, respectively. From 2012 on, she is a postdoctoral research fellow of the Flemish research fund (FWO) at the Department of Mechanical Engineering, KU Leuven. From 2010 to 2011, she was a visiting PhD scholar at the computational biomechanics lab of Stanford University. Her research interests include nonlinear finite element modeling of biological soft tissue, experimental characterization of material properties of cardiovascular tissue, damage detection and quantification in cardiovascular tissue and computational aspects of robotic surgery. She has (co-)authored 20+ international publications and is currently supervising multiple $\mathrm{PhD}$ and master thesis students.

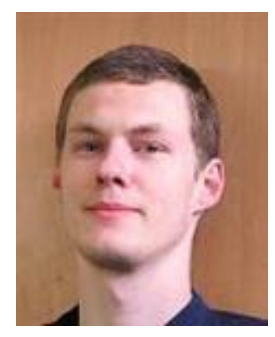

Michal Böhm works at Materialise since 2003. Started in company as Vacuum casting teamleader, then Vacuum casting (VC) specialist, Production Manager, Research Engineer, Product manager. He works on research for new materials, and new VC techniques. His research interests include improvement of Vacuum casting manufacturing techniques, and complex shapes manufacturing.

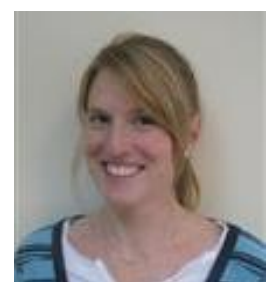

Dr. Ir. Eva Verhoelst received her MSc and PhD degree in Bioengineering at KU Leuven, Belgium, in 2003 and 2011, respectively. From 2013 on, she is research project manager at Materialise NV, managing several internally and externally funded medical research projects. Her research interests include image processing, cardiovascular intervention planning, and medical device design. She has (co)authored 10+ international publications and conference proceedings, and is currently supervising multiple $\mathrm{PhD}$ students. 